\title{
Calidad educativa, a propósito de la gestión comunitaria en nueve establecimientos educativos de Medellín: 2010 a 2013
}

Quality of Education Regarding Community

Management in Nine Educational

Establishments of Medellín: 2010 to 2013

Qualidade educacional em relação com a gestão comunitária em nove instituições educativas de Medellín: 2010 a 2013

\author{
Jose Wilmar Pino Montoya** (D) orcid.org/0000-0001-9035-2058 \\ Melina Castaño Gómez*** (iD) orcid.org/0000-0002-2354-4014
}

doi: $10.17227 /$ rce.num77-4925

Para citar este artículo: Pino, J. y Castaño, M. (2019). Pertinencia y actualidad de la pedagogía amigoniana. Revista Colombiana de Educación, 77, doi: 10.17227/rce.num77-4925.

\section{(c) $(1) \Theta$}

* En esta investigación participaron activa y colaborativamente diferentes instituciones, entre las que se cuentan la Secretaría de Educación de Medellín, Proantioquia, Universidad de Antioquia, Universidad Pontificia Bolivariana, la Universidad San Buenaventura, Seccional Medellín, la Fundación Cinde y la Fundación Universitaria Luis Amigó.

** Doctor en Filosofía por la Universidad Pontificia Bolivariana. Docente de la Universidad Católica Luis Amigó, Colombia. Correo electrónico: jose.pinomo(amigo.edu.co

*** Estudiante del último semestre del pregrado en Ciencia Política. Universidad de Antioquia Auxiliar de 


\section{Resumen}

El objetivo de este trabajo es reconocer las concepciones de la gestión comunitaria, las prácticas asociadas con esta y su impacto en el mejoramiento académico-pedagógico de los establecimientos educativos participantes. En esta investigación se privilegió el estudio cualitativo en perspectiva de una hermenéutica comprensiva. Se hicieron nueve entrevistas a directivos y docentes de nueve establecimientos educativos, incluidos públicos y privados, de Medellín que hubieran participado de los premios Ciudad de Medellín a la Calidad de la Educación. Se encontró que la gestión comunitaria de los establecimientos investigados se centró en su relación con el contexto, la proyección a la comunidad, las relaciones interinstitucionales, el seguimiento a egresados y la prevención del riesgo. A modo de conclusión, se resalta que los establecimientos educativos participantes en los premios fortalecen su gestión comunitaria a través de las alianzas con otras organizaciones, lo que afianza la construcción del tejido social y contribuye a mejorar su gestión educativa.

\section{Palabras clave}

educación; evaluación educativa; política educativa; mejora educativa; educación secundaria

\section{Keywords}

education; educational assessment; educational policy; educational improvement; secondary education

\begin{abstract}
The purpose of this work is to recognize the conceptions of community management, the practices associated thereto, and their impact on the academic-pedagogical improvement of the participating schools. In this research, privilege was given to the qualitative study in perspective of a comprehensive hermeneutics. Nine interviews were carried out with executives and teachers from nine schools-both public and private-in Medellín that participated in the Ciudad de Medellín awards for Quality in Education. It was found that the community management of the researched schools focused on their relationship with the context, the projection to the community, the interinstitutional relations, the follow-up on graduates and risk prevention. By way of conclusion, it is highlighted that the schools that participated in the awards strengthen their community management by forming alliances with other organizations, which strengthens the construction of the social fabric and contributes to improving their educational management.
\end{abstract}

\section{Resumo}

O objetivo deste trabalho é reconhecer as concepções da gestão comunitária, as práticas associadas com esta e seu impacto no aprimoramento acadêmico-pedagógico das instituições educativas participantes. Nesta pesquisa privilegiamos o estudo qualitativo em perspectiva de uma hermenêutica compreensiva. Realizamos nove entrevistas a diretivas e professores de nove instituições educativas, públicas e particulares, de Medellín, que participaram dos prêmios Cidade de Medellín à Qualidade da Educação. Encontramos que a gestão comunitária das instituições pesquisadas está centrada em sua relação com o contexto, a projeção à comunidade, as relações interinstitucionais, o seguimento a ex-alunos e a prevenção do risco. Como conclusão, assinalamos que as instituições educativas participantes dos prêmios fortalecem sua gestão comunitária através das alianças com outras organizações, afiançando a construção do tecido social e contribuindo para o aprimoramento de sua gestão educacional.

\section{Palavras-chave}

educação; avaliação educacional; política educacional; aprimoramento educacional; educação secundária 


\title{
Introducción
}

Desde sus inicios, en el 2007, los premios Ciudad de Medellín a la Calidad de la Educación han pretendido reconocer la gestión de los establecimientos educativos: públicos y privados, y de sus actores (docentes y directivos), en pro de la búsqueda de la calidad y el mejoramiento de la educación. Es por ello que, en el acuerdo municipal 26 del 2007, se describe el premio como "un espacio de encuentro ciudadano en torno a la educación [que pretende] reconocer y destacar los procesos y los resultados en materia de mejoramiento de la calidad educativa en las instituciones educativas oficiales y privadas del Municipio de Medellín". Así mismo, en el acta de compromiso del 2014, se advierte que "este reconocimiento institucional instituye la calidad educativa y los esfuerzos adelantados como un aspecto central de las dinámicas y las lógicas escolares" (Secretaría de Educación de Medellín [sem] et al. 2014, p. 1).

Estos premios a la calidad educativa, desde su institucionalización, han realizado diferentes entregas en sus múltiples modalidades, que han generado en las instituciones participantes y sus actores, diferentes ejercicios de reflexión, construcción y acción que sería relevante rescatar para determinar lo que ha sucedido en las instituciones, a partir de su participación en los Premios Ciudad de Medellín a la Calidad Educativa. En el marco de estos premios, la Secretaría de Educación de Medellín se propuso:

\begin{abstract}
Materializar una alianza público-privada donde se aúnen esfuerzos para adelantar un proceso de investigación alrededor del Premio a la Calidad de la Educación Ciudad de Medellín, y sus contribuciones al mejoramiento de los establecimientos educativos a través del análisis crítico, la sistematización, realimentación y difusión de sus experiencias significativas y construcciones escolares. (SEM et al., 2014, p. 2).
\end{abstract}

Teniendo en cuenta, entonces, el propósito de los Premios Ciudad de Medellín a la Calidad de la Educación se adelantaron seis investigaciones, entre las cuales se encuentra el presente estudio denominado: "Gestión comunitaria en el marco de la convocatoria 'Maestros para la vida' de la ciudad de Medellín entre los años 2010-2013", realizado por el grupo de investigación Educación, Infancia y Lenguas Extranjeras de la Universidad Católica Luis Amigó. Este proyecto de investigación se propuso evaluar e indagar el impacto y las eventuales problemáticas que han tenido los Premios Ciudad de Medellín. De este modo, el estudio tiene por objetivo reconocer las concepciones de la gestión comunitaria, las prácticas asociadas y su impacto en el mejoramiento académico-pedagógico de los establecimientos educativos participantes. A partir de lo anterior, la investigación orientó sus objetivos a la descripción de las diferentes estrategias utilizadas por los establecimientos educativos, en lo que respecta a la gestión comunitaria para

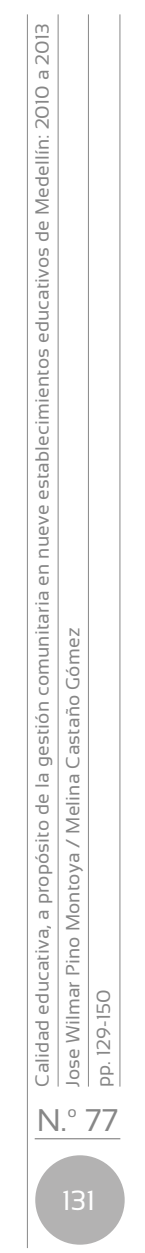


el fortalecimiento y enriquecimiento de la gestión escolar; a la identificación de los diferentes escenarios y las formas de comunicación utilizadas para estimular la participación de los miembros de la comunidad educativa y, por último, a la caracterización de las propuestas en torno a los premios a la calidad que dan cuenta de las mejoras en el ámbito académico de los establecimientos sujetos del estudio. De esta manera, se busca apoyar lineamientos que contribuyan al fortalecimiento de la gestión comunitaria coherente con el contexto, en cuanto que la comunidad desempeña un papel decisivo en la elaboración de propuestas y en la discusión y definición de prioridades en el ámbito escolar.

Esta investigación aporta conocimientos sobre lo realizado en los establecimientos educativos públicos y privados de la ciudad, su contexto inmediato, la labor que han realizado en la comunidad y cómo esta posibilita el mejoramiento de los procesos pedagógicos de los maestros y maestras de dichas instituciones, lo que redundaría en el fortalecimiento de los procesos educativo para lograr mejor y mayor calidad educativa en la ciudad.

Por otra parte, los resultados de la investigación son un insumo relevante que orientaría la reformulación de proyectos educativos de gestión y generación de políticas públicas. Del mismo modo, son una herramienta para optimizar los programas que pretendan mejorar las condiciones de los establecimientos educativos y de su entorno comunitario. Es decir, podrá servir de marco de referencia para que las instituciones educativas, la Secretaría de Educación de Medellín, las entidades públicas y privadas y las ONG fortalezcan las políticas, programas y proyectos educativos que tengan como fin lograr la calidad de la educación y el desarrollo de la ciudad.

Acorde a lo anterior, el presente artículo se enfoca en describir la relación que tienen los establecimientos educativos de Medellín con la gestión comunitaria. Dicha gestión se basa en tres componentes: el primero, se refiere a la pertinencia al contexto, proyección a la comunidad y relaciones interinstitucionales; en el segundo componente, se cuentan los egresados, y, en el tercero y último, la prevención del riesgo.

\section{Referentes teóricos}

\section{Gestión comunitaria}

Para comenzar esta conceptualización sobre la gestión comunitaria y su relación con la gestión educativa, es pertinente referirse primero al concepto de comunidad, como la define Avella (2004): 
Como un conjunto de representaciones en lo estructural, simbólico, comunicacional y físico, en donde los individuos se relacionan de manera permanente compartiendo sus narrativas. Este estilo asume la comunidad como protagonista del saber y del conocimiento que emerge de las prácticas comunitarias. (pp. 30-31).

El anterior concepto, necesariamente, debe llevar a la búsqueda de nuevos criterios para lograr la calidad educativa y dinamizar su gestión a través del fomento de la participación activa de la comunidad, la misma que "debe entenderse como la construcción progresiva de autonomía en los miembros de la comunidad educativa en un proceso complejo y permanente que se constituye en un medio y en un fin de la educación" (Sierra, Serna y Pérez, 2002, p. 21). La gestión de la comunidad, además, orienta y sienta las bases para diseñar la construcción del manual de convivencia pertinente y "acordados por los agentes de la institución, los resultados de las evaluaciones, los proyectos transversales y el contexto de la institución" (Martínez et al., 2012, p. 20). En este sentido, la gestión de la comunidad "se encarga de las relaciones de la Institución con la comunidad, de la participación y la convivencia" (Ministerio de Educación Nacional, 2010, p. 157). De allí que las instituciones educativas, en procura de consolidar, alcanzar y buscar la calidad, deben tener presente que

[...] los procesos de participación en la comunidad educativa deben cualificarse progresivamente, a partir de la habilitación de los diversos actores que la conforman con nuevas comprensiones y actitudes democráticas que permitan simultáneamente alcanzar la calidad de la educación y la construcción de una sociedad plural y justa. (Sierra et al., 1999, p. 25).

Es en este punto donde resulta importante las sugerencias que hace la Alcaldía de Medellín (2013), cuando advierte que la institución educativa, en cuanto referente social y cultural de su entorno, define, mantiene y hace seguimiento a la interrelación que mantiene con su medio social y productivo donde está inscrita. Tal interrelación se materializa en los siguientes tres componentes: la pertinencia el contexto, proyección a la comunidad y las relaciones interinstitucionales; cuando se hace referencia a este componente se hace alusión, según la Alcandía de Medellín:

[...] al conjunto de estrategias y alianzas que realizan los Establecimientos Educativos con base en el reconocimiento del contexto. La escuela revisa permanentemente su relación y participación en la construcción del tejido social y su accionar como protagonista del desarrollo local y nacional. Asimismo, busca la articulación con entidades públicas y privadas que ofrecen servicios complementarios para el fortalecimiento de las competencias y habilidades de los estudiantes e involucra a la familia como aliado estratégico. (Alcaldía de Medellín, 2014, p. 22). 
Un segundo componente de la gestión comunitaria es el seguimiento a los egresados. Para fines de esta investigación, el egresado se entenderá como lo interpreta la Organización para la Cooperación y el Desarrollo Económicos (OCDE):

[...] el concepto de egresado guarda correspondencia con el de graduado. Define como graduado a la persona que completó exitosamente todos los requisitos de un programa educativo. Señala que la terminación exitosa de un programa implica la demostración, por parte del estudiante, de las habilidades y conocimientos adquiridos, pudiendo ser a través de un examen o serie de exámenes, o bien acumulando el número específico de créditos del programa, de tal forma que pueda obtener la acreditación de los estudios realizados. El graduado debe ser contabilizado en el año en que completó todos los requisitos del programa. (OCDE, citado por UNAM, 2006, p. 13).

Por ello, y para el Premio Ciudad de Medellín a la Calidad Educativa, es importante tener en cuenta al egresado porque contribuye a la proyección social del establecimiento educativo.

En este sentido, es importante hacerle seguimiento para contrastar sus desempeños académicos, laborales y sociales con la propuesta educativa institucional para realizar los ajustes necesarios de acuerdo a las demandas de la sociedad actual. A su vez, vincularlos activamente a la vida institucional. (Alcaldía de Medellín, 2014, p. 22).

El tercer y último componente que tiene presente la gestión comunitaria es el de la prevención de riesgo, la cual "se refiere a las estrategias que establece y realiza la institución educativa para minimizar los riesgos naturales, físicos y psicosociales que pueden afectar las dinámicas propias de la misma" (Alcaldía de Medellín, 2013, p. 28).

Para cumplir con este componente el establecimiento educativo debe

\begin{abstract}
Describir las prácticas para detectar, prevenir y mitigar los riesgos naturales, físicos y psicosociales que tiene definidas el establecimiento para implementar el Plan de Gestión Escolar del Riesgo; exponer los vínculos intersectoriales y sus correspondientes acciones para promover una cultura del autocuidado $y$, presentar evidencias de la participación de los miembros de la comunidad educativa en esta. (Alcaldía de Medellín, 2014, p. 35).
\end{abstract}

En conclusión, se podría decir que una institución educativa que busque alcanzar la calidad estaría encaminada a lograr la participación de la comunidad, es decir, una gestión de la comunidad, fundamentada en la pertinencia de sus acciones, adecuadas e inspiradas en su contexto, "su proyección a la comunidad y sus relaciones interinstitucionales con 
el entorno más cercano al área de influencia. Por otra parte, se interesa por el seguimiento a egresados y la prevención de riesgos" (Alcaldía de Medellín, 2012, p. 13).

Definido el concepto gestión comunitaria y sus componentes, es imperante además comprender cómo se une este componente con el de calidad y, para ello, se hace preciso también centrarse un poco en el concepto de calidad educativa.

\section{Conceptualización de calidad educativa}

En este punto, se puede precisar que el concepto calidad educativa puede ser estudiado, como lo dice Vásquez (2013) desde tres enfoques: el tradicional, el moderno y el dialéctico. El tradicional "...remite a la concepción esencialista donde el ente o proceso de calidad ha desarroIlado intrínsecamente sus caracteres" (p. 64). Una segunda definición está inspirada en la relación entre recursos, procesos y fines; según los argumentos emitidos por Vásquez (2013), este tipo de calidad educativa está más relacionada con la eficacia y eficiencia en procesos y medios para el logro de unos fines previamente establecidos. Aquí, un programa de calidad sería aquel que cuente con los recursos necesarios y, además, que los emplee eficientemente en los procesos educativos. Por último, el concepto de calidad, desde la perspectiva dialéctica se refiere a:

a) transformar un bien o servicio; b) criticar el conocimiento y elaborar, innovar y crear conocimientos; c) interactuar críticamente con sus alumnos; d) la racionalidad educativa basada en la historicidad; e) los alumnos deben ser autodidácticas, investigadores, críticos, creativos e innovadores. (Vásquez, 2013, p. 64).

Es de aclarar que la explicación de la calidad educativa no se agota solamente en estas tres características, es decir, estas no explican con suficiencia el concepto aquí estudiado, se hace necesario, por tanto, incluir otros factores en los que necesariamente no deben estar comprometidos solo los directivos de las instituciones, sino que se deben sumar otros actores, como los profesores.

Por otro lado, y en el marco de la educación, Palma (2008), citando a Mortinore (1991), señala:

La escuela de calidad es aquella que promueve el progreso de los estudiantes en una amplia gama de logros intelectuales, sociales, morales y emocionales, teniendo en cuenta su nivel socioeconómico, su medio familiar y su aprendizaje previo. Un sistema escolar eficaz maximiza las capacidades de las escuelas para alcanzar estos resultados. Lo que supone adoptar la noción de valor añadido en la eficacia escolar. (Mortinore, citado en Palma, 2008, pp. 3-4). 
En este sentido, se puede argumentar que "la calidad educativa es entendida de forma multidimensional y contextual. Más allá de los índices de matrícula, retención y aprovechamiento académico [...] debe entenderse el carácter multideterminado y dinámico de la calidad" (Rodríguez, 2010, p. 18).

Según lo argumentado anteriormente, son muchas las acepciones del concepto calidad educativa, no obstante, es pertinente señalar que, para los fines propuestos en este trabajo, se toma una definición de calidad educativa que permita ser explicada y que esté orientada al desarrollo del ser humano de manera integral. Una perspectiva útil en este sentido es la de Ramírez y Salinas:

Una educación con calidad debe fomentar la solidaridad, el sentido de justicia, la tolerancia, el respeto por los demás, hábitos de mejoramiento y superación personal y el desarrollo de un razonable equilibrio emocional y mental. Así mismo una educación con capacidad, a través del currículo de valorizar y hacer suyas su forma de pensar y sentir que una comunidad tiene para enfrentar y darle sentido a la vida cotidiana. (Ramírez y Salinas, citados por Jaramillo, 1999, p. 97).

Se puede concluir, entonces, que la calidad educativa es un proceso continuo de perfeccionamiento y mejoramiento de las instituciones educativas, que buscan, como fin último, "responder eficientemente a las necesidades de los individuos, entendiendo al mismo tiempo que ella debe inscribirse en las necesidades colectivas que tiene la escuela, la colectividad y la sociedad" (Jaramillo, 1999, p. 97).

\section{Metodología}

Para esta investigación se privilegió la investigación cualitativa, en perspectiva de una hermenéutica comprensiva. Este método permite comprender la experiencia de las instituciones educativas (públicas y privadas) postuladas, nominadas y ganadoras de los premios a la calidad de la educación en la ciudad de Medellín.

En el marco de esta metodología, se utilizó la entrevista en profundidad como instrumento privilegiado para la recolección de la información. Esta técnica facilitó la expresión espontánea de experiencias individuales y grupales, que suministraron suficiente información sobre el tema propuesto en la investigación. Para la aplicación de las entrevistas, se invitaron a docentes y directivos docentes de las instituciones educativas participantes del Premio Ciudad de Medellín a la Calidad de la Educación durante los años 2010 y 2013, seleccionados teniendo en cuenta varios criterios entre los que se encontraban el ser participantes provenientes tanto de la categoría de los postulados, de los preseleccionados y de los ganadores. 
Por otra parte, fue importante que la población se escogiera de todos los lugares de la ciudad; que hubiera representación de los distintos núcleos educativos; además, se tuvo presente la equidad de género, es decir, participación masculina y femenina en los premios.

Sobre los establecimientos que participaron de la investigación, se puede decir que se eligieron 9 instituciones entre públicas y privadas que cumplieran los siguientes criterios: 3 instituciones postuladas, pero que no fueron nominadas; 3 instituciones que se presentaron y fueron nominadas; y 3 ganadoras en alguna de sus versiones. Estas instituciones debieron haberse presentado a los premios durante el año 2010 y 2013.

\section{Hallazgos y discusión}

\section{Concepciones sobre la gestión comunitaria}

Respecto al concepto gestión comunitaria recabado en las entrevistas realizadas a los directivos y docentes, se puede decir que, si bien este no se encuentra expresado de manera clara en los testimonios, sí se evidencian algunos datos que dan cuenta de cómo se gestiona este componente en los diferentes establecimientos educativos (EE) y de los cuales se puede deducir la concepción de los indagados al respecto.

Para comenzar este aparte, uno de los participantes presenta un testimonio que ofrece un concepto de gestión comunitaria acorde con un proceso en el que los eE no solo se limitan a diagnosticar o describir los problemas de la comunidad. Es además relevante que la gestión integre actividades que ayuden y que tengan como fin el desarrollo humano de los integrantes. Así lo evidencia el siguiente argumento de uno de los participantes:

La comunidad se ha visto impactada porque si bien nosotros Ilevábamos a nuestras estudiantes a unas practica pedagógicas en diferentes instituciones ahora entonces no solamente estamos vinculando esas otras instituciones con el acompañamiento, con la recreación, sino con estos proyectos de investigación. Ahora ellos son participes porque le están haciendo seguimiento a esta propuesta de investigación que tenemos y de formación de líderes a partir de ello. (Entrevista 3).

En este aparte se puede observar que la concepción de la gestión comunitaria se relaciona con la de brindar alternativas pedagógicas, recreativas e investigativas en conjunto con otras instituciones con el fin de potenciar en los integrantes de la comunidad unas competencias, en este caso, la de formar líderes que luego tendrán la tarea de formular alternativas conjuntas para solucionar los problemas. 
De este modo se hace necesario identificar la

[...] gestión comunitaria como un proceso educacional en donde el agente propulsor no se dedica solamente a identificar, diagnosticar y dar recomendaciones para la solución de problemas, sino que conduce a que mediante acciones educativas la comunidad se organice, defina necesidades formule planes y ejecute actividades que la conduzcan a elevar su nivel de vida en forma continuada. (Institución Educativa Chachagüí, s. f., párr. 1).

Como se observa la concepción de los entrevistados sobre la gestión comunitaria no está lejos del anterior argumento, en cuanto que en sus testimonios se observa un importante involucramiento del eE con la solución de problemas reales que vive la comunidad en la que se integra.

Entonces hemos generado incluso alguna posibilidad de empleo, y lo otro, con las alianzas, un poco el tema del premio a la excelencia que se ganó el año pasado el colegio Montessori es un poquitico de nosotros porque tenemos un convenio con este colegio donde ellos trabajan con los papás, los docentes y los niños haciendo un acompañamiento con un proyecto que se Ilama "PAs", entonces se puede ver cómo esas estrategias y esos convenios siguen visibilizando estas comunidades donde trabajamos, más que la escuela es la comunidad como tal. (Entrevista 11).

Según el anterior testimonio, la gestión comunitaria se relaciona con la implementación de actividades que mejoren la calidad de vida del entorno en el que participa la institución. Por ello, "exige la plena participación de la escuela en asuntos colectivos, única manera de socializar al niño mediante el manejo de problemas reales y por otra parte de vitalizar la acción escolar, despertando y fomentando una decidida protección hacia ella" (Jiménez, 1997, p. 34).

Otro concepto sobre gestión comunitaria que se visualiza en los testimonios es el que está relacionado con lo que dicen Mamani y Machicado (2012):

Desde la gestión educativa comunitaria, la relación de la institución educativa con la comunidad o la sociedad, debe aportar con ideas para proyectos conjuntos dirigidos al mejoramiento de la calidad de vida, así como también el avance de la comunidad educativa hacia la inclusión, participación, convivencia armónica, y proyección hacia el futuro. (párr. 11).

El testimonio que recrea y sustenta lo dicho anteriormente es el expresado por en la entrevista 7: 
La institución está ocupada todo el tiempo, la tercera edad, grupo juvenil, de baile. Este año un grupo que tenemos acá que nos ocupa ciertos salones en la noche de música y de artes. El programa de medio ambiente nosotros hemos identificado ancianatos, lugares donde se puede arborizar, tenemos los días sábados con adultos de la comunidad va dirigido a adultos mayores de 18 años de la comunidad, sea que tengan su hijo aquí o no tengan su hijo acá, el programa está dirigido a la enseñanza del inglés, un programa de reflexión acerca del medio ambiente e informática para adultos.

Lo relevante de estas experiencias es que los diferentes testimonios exponen unos significados de la gestión comunitaria teniendo presente lo que afirma la Alcaldía de Medellín (2014), que los establecimientos educativos de la ciudad demuestran un amplio compromiso con su comunidad y son responsables del mejoramiento de su entorno asumiendo un compromiso con su comunidad y desplegando sus propuestas educativas, formativas y pedagógicas en el apoyo al desarrollo de los otros (comunidad), sin abandonar el rigor que ha de caracterizar su existencia y sin caer en el artificio de responsabilizarse o dejarse responsabilizar de la crisis o de la solución.

\section{Pertinencia al contexto, la proyección a la comunidad y las relaciones interinstitucionales}

En lo que se refiere a la pertinencia al contexto, se demuestra en los datos encontrados que existe una importante pertinencia de la institución, con relación al contexto que se explica en habitabilidad de los alumnos cerca de la institución una de las entrevistas advierte: "Calasanz para todos, es una actividad donde el colegio presta ayuda de refuerzo a chicos de aquí de la comunidad 13" (Entrevista 1). Este argumento se complementa con el siguiente: "La acción comunal viene habla con nosotros, se estudia la posibilidad y si es viable se hace, es más como la disposición de atenderlos, que de pronto no se den" (Entrevista 1).

Este componente lo complementa el siguiente testimonio:

[...] el colegio se ha caracterizado por ser un colegio del barrio, usted viene un sábado o un domingo aquí y aquí hay más gente, aquí hay más gente los sábados y domingos que de lunes a viernes, porque aquí hacen curso de todos, aquí lo prestan para todo lo que la comunidad necesita, que hay mucha comunidad catecumenos y aquí hacen todos sus encuentros, pues aquí hacen todos sus eventos. (Entrevista 9).

En la entrevista 11 también se puede visualizar este componente de la gestión comunitaria y cómo la institución fortalece su pertenencia al contexto con la estrategia de tener presente sus necesidades y demandas 
"[...] las estrategias para acceder a la comunidad y mencionábamos los centros de interés, entonces invitamos a la comunidad, pero con cosas que les sirven realmente a partir de las necedades que tienen $[\ldots]^{\prime \prime}$.

Como se observa, para fortalecer su pertinencia al contexto, los EE tienen la capacidad de leer las situaciones y necesidades de su entorno para proponer desde allí diferentes alternativas que solventen sus dificultades, por ello, las EE tienen la capacidad de

[...] explicar cómo, la oferta de proyectos formativos complementarios, el uso de la biblioteca, de recursos informáticos, y de los demás espacios institucionales y el desarrollo de actividades artísticas, culturales, deportivas, académicas, son aprovechados por la comunidad del sector como espacio para compartir, complementar saberes y experiencias que nutren a su vez el proyecto educativo institucional. (Alcaldía de Medellín, 2013, p. 27).

Un primer testimonio que da cuenta de este recurso de los Ee es el siguiente: "Calasanz para todos, es una actividad donde el colegio presta ayuda de refuerzo a chicos de aquí de la comunidad 13" (entrevistado 1). Además, agregan que "el gran aporte de la gestión comunitaria es por un lado que fortalece estructuras familiares, en todo lo que decíamos ahora, estilos de vida saludables [...]" (entrevistado 1). En este mismo sentido, se agrega que la "proyección social la tenemos en la vereda Granizal, sector Altos de Oriente. Íbamos a llevarles mercados los sábados con los padres de familia y alumnas de Calasanz, y con las niñas del Colegio León XIII" (Entrevista 2).

Otros EE de la ciudad utilizan como estrategia para proyectarse a la comunidad la alianza con otras instituciones, el siguiente testimonio evidencia la realización de esta alternativa:

[...] resulta un tema de investigación o una propuesta desde Explora o Proantioquia y nosotros siempre participamos, siempre nos postulamos, siempre queremos mostrar lo que hacemos. Sí, es que la Yamaha dijo, el proyecto de ustedes, venga hermano que eso está muy bueno allá, entonces vienen trabajando con nosotros, entonces nos puso un profesor, $y$ ahí vamos creciendo con la Yamaha. (Entrevista 6).

Las diferentes formas de gestión comunitaria pasa también por combinar la teoría con la práctica.

El modelo de la alternancia, es un modelo francés, que viene pues a darnos unas guías, unas luces de cómo se puede alternar lo teórico con lo práctico, pero es algo que constantemente se hace en la Normal, pues eso constantemente se está poniendo en escena, la teoría con la práctica en todo momento se hace, acá le dimos un enfoque mayor porque ellas son mujeres con muchas dolencias personales y falta de 
formación de autoestima, entonces primero había que hacer un trabajo de fortalecimiento y el estudio les da a ellas la posibilidad de avanzar más y valorarse, más frente a lo que hacen. (Entrevista 4).

En la entrevista 7 se encontró que las actividades de proyección a la comunidad también acogen a mayores de 18 años ajenos a la comunidad educativa. Dice así el testimonio: “Tenemos los días sábados con adultos de la comunidad mayores de 18 años, sea que tengan sus hijos aquí o no tengan, el programa está dirigido a la enseñanza del inglés, la reflexión acerca del medio ambiente e informática" (Entrevista 7).

El hallazgo principal relacionado con las relaciones interinstitucionales es la capacidad que tienen los Ee para construir alianzas con otras instituciones. Es de destacar, además, que el número de alianzas que maneja cada institución es alto, en promedio 4,8 alianzas por EE; la institución más proactiva en este aspecto cuenta con 12 alianzas. Un ejemplo de esta situación se encontró en la entrevista 2:

La construcción del tejido social no solamente se da desde las instituciones externas (Inder y la Universidad de Cambridge), sino desde este hacia la comunidad, como se evidencia a través de los Scouts, del proyecto Calazans para todos (enseñanza a población de la comuna 13), servicio social que se presta a las diferentes instituciones (centro geriátricos, centros de salud, bibliotecas, entre otras) que finalmente logren establecer convenios y generar la ayuda suficiente que la comunidad requiere y que la comunidad académica pueda generar.

La anterior experiencia la complementa uno de los entrevistados, quien dice: "En el servicio social se hacen convenios con instituciones educativas cercanas, pueden ser hogares geriátricos, pueden ser jardines infantiles, pueden ser hospitales" (Entrevista 1).

En otros EE, las alianzas y las relaciones más relevantes se establecen con la comunidad. Un testimonio que da cuenta de este tipo de experiencia es el siguiente:

La pertinencia social en dicha institución educativa, se hace relevante puesto que la comunidad interactúa directamente con la escuela, desde la historia, la comunidad creo la escuela para bien de todos y esto hace que docentes, estudiantes, padres de familia y directivos, hablen un lenguaje común. El tejido social aborda las necesidades tanto de la escuela como de la comunidad, así entrelazan con actividades culturales y deportivas el bienestar de todos, de esta manera la relación con las juntas de acción comunal, iglesias, secretarías de salud, de tránsito, Inder y otros programas hacen que sea eficiente y brinde buenos resultados. (Entrevista 5). 
La comunidad, entonces, se constituye en uno de los principales aliados de los establecimientos educativos para llevar a cabo sus propuestas y proyectos, lo cual sirve de soporte para el desarrollo de diferentes actividades.

Si bien el entorno más inmediato se convierte en un socio estratégico para que los EE, estos acuden también a alianzas con instituciones internacionales. La siguiente experiencia soporta el anterior argumento:

La comunidad en general permitió cambios, y la IE pensando en el contexto, se dio a la tarea de crecer de manera competente, Ilevando a los estudiantes a permanecer en la academia por medio de la participación de proyectos y eventos educativos como: Pentacidad que permitió formar en valores por dentro y por fuera de la escuela, pues la institución se convirtió en red, en ejemplo para otros colegios aledaños que ayudan a toda la comunidad. (Entrevista 5).

Es de resaltar entre las alianzas más importantes que realizan los establecimientos públicos y privados, las que hacen con las instituciones de educación superior de carácter internacional. "La institución se destaca por sus alianzas [...] internacionales, como la [construida] con FS Barcelona [...] Universidad de Texas" (Entrevista 1).

Además, se destacan las alianzas con diferentes universidades locales. En este sentido se pronuncia en la entrevista 4:

Con Eafit en este momento la formación de mujeres esta con Eafit un convenio y el [...], otro de los convenios con San Buenaventura, tenemos convenio con la Universidad de Antioquia, ah y de bienestar también tenemos la parte de los médicos que vienen y hacen intervención con los muchachos que son ginecólogos [...].

Entre las diferentes alianzas, se cuentan además las que se realizan con los entes gubernamentales: "Tenemos alianzas con Secretaría de la Mujer, con el municipio de Sabaneta, con el municipio de Necoclí, con el corregimiento de Doradal en Puerto Triunfo" (Entrevista 11). En la entrevista 10 se expresa al respecto: "Hoy estamos con la Secretaría de Educación y con la Vicealcaldesa de Educación y Cultura montando el día de la camiseta para toda la ciudad".

En resumen, se podría decir que la mayoría de alianzas se establecieron a nivel local, si bien hay algunas instituciones que funcionan en varios países como Terpel, Yamaha, Sisco y Cruz Roja, fueron con filiales locales con quienes los EE establecieron contacto. Solo uno ha establecido relaciones internacionales, específicamente con FS España, FBC, Imagine y la Universidad de Texas; además, una de sus sedes es fruto de cooperación internacional con organizaciones de Puerto Rico. La institución con la que más se establece alianzas es con la Secretaría de la Mujer. 


\section{Egresados}

Si bien la categoría habla de la importancia de los egresados, puesto que contribuyen a la proyección social de los EE. Es fundamental hacer seguimiento a la función que tienen ellos en el contexto, de acuerdo con esto, los EE de la muestra han establecido algunos parámetros para poder cumplir con dicho requisito, pero sigue siendo el punto de quiebre de las instituciones, puesto que es complicada la recolección de información y su seguimiento.

No obstante, y a pesar de la falta de información al respecto y de las pocas actividades realizadas en la investigación, se aprecia que el poco trabajo que hacen las instituciones con los egresados consiste en mantener información relacionada con el acceso de los graduados a las instituciones de educación superior, las reuniones de integración con egresados, las jornadas deportivas y eucaristías. Por otra parte, algunos establecimientos educativos manifiestan que han logrado un alto sentido de pertenencia en sus egresados, hasta tal punto que varios de ellos llegan a realizar labores con los mismos estudiantes de la institución. Lo anterior se evidencia en el siguiente testimonio:

¿Acá? Una belleza, ¿sabe qué hace el de la Universidad de Antioquia? Se viene todos los domingos, para los estudiantes de acá que estén teniendo problemas con matemáticas [...]. Entonces él viene y les explica. No cualquiera lo hace y gratis [...]. (Entrevista 7).

Asimismo, dice el entrevistado: "Tenemos algunos egresados de acá de la institución, que nos asesoran, pues ellos trabajan en los bancos, y ellos vienen acá a darnos todas las asesorías que necesitamos para hacer prestamos, todo eso" (Entrevista 7). El representante de esta misma institución, además, argumenta: "Y la muchachita que está estudiando en preescolar, ya está haciendo la práctica acá, espectacular. Entonces los mismos estudiantes tienen eso, porque se les ha enseñado eso, la gratitud" (Entrevista 7).

En este mismo sentido se manifiesta:

El sentido de pertenencia es alto y hay muchos egresados que actualmente por sus cargos, por sus trabajos, por sus empresas, vincularlos con la institución mejora mucho las dinámicas del mismo colegio, en abordar proyectos, posibilidades de recursos, posibilidades de mejorar en sus trabajos de proyección social y es un potencial que lo hemos aprovechado al $100 \%$. (Entrevista 2).

En la poca información que se encontró en los eE relacionada con los graduados sobresalen instituciones que tienen una práctica innovadora en el trabajo con estos, la cual consiste en formar y certificar sus competencias. 
En capacitaciones tenemos tres momentos en el año: uno se invitan junto con una persona del SENA para que certifiquen sus competencias, eso es fundamental y es muy difícil porque de todos nuestros egresados solo 15 han certificado competencias y es un logro grandísimo que puedan certificarse a partir de la técnica que hicieron. (Entrevista 4).

Y agregan que en formación tienen el cine foro anual con el interés de ellos y se invita a su participación; hay una reunión formativa y fundamentalmente lo enmarcamos en el tema de certificación de competencias o mostrarles cuál es la ruta laboral que en este momento hay en la ciudad; se les informa dónde están las canastas para el empleo; a dónde pueden llevar las hojas de vida, y se les indica cómo diligenciarlas.

Con relación a la estrategia para mantener informados a los egresados, dice el mismo entrevistado:

Hay muy buen contacto, ya la respuesta es otra cosa, a veces es buena, a veces no, depende de muchos factores, pero normalmente hay una muy buena relación con los egresados, suelen pasar solicitando préstamo de sala de internet y en el Facebook hay una persona que es la dinamizadora cultural que es la encargada de montar los eventos de la escuela y ellos continuamente están ahí. (Entrevista 4).

Según los datos que arrojan las entrevistas, se concluye que hay muy poco trabajo con el grupo de egresados. Se puede apreciar que son varias las instituciones que no tienen ningún tipo de programa dirigido a esta población.

\section{Prevención del riesgo}

Los resultados que arrojaron las entrevistas muestran que son múltiples las actividades realizadas, lo anterior ligado a las necesidades propias de cada eE y del contexto en el que están ubicados, en concordancia con su visión y misión.

La primera de estas actividades enfatiza su quehacer en la prevención del riesgo psicosocial, desarrollando actividades manuales y deporte. Así lo hace saber el siguiente testimonio:

La institución cuenta con espacios de intervención en arte y deportes, donde se logra la participación de la comunidad y así evitar el ingreso de los estudiantes a contextos segregados y no favorables, puesto que la drogadicción y el consumo continuo de sustancias psicoactivas [...]. (Entrevista 2).

A esta estrategia se le suma y es complementada con las actividades realizadas con los mismos maestros: 
La prevención de riesgos es importante trabajarlo también con los maestros, puesto que son ellos quienes proporcionan el aprendizaje y la estabilidad de una institución, de esta manera se invitan a los juegos y se les está habilitando un gimnasio [...]. (Entrevista 2).

En otras circunstancias, los directivos y docentes optan por actividades más espirituales y de expresiones verbales. Para más claridad sobre este asunto dice una de las participantes:

Los retiros anuales que se realizan para compartir con las estudiantes, traen consigo otros vínculos y haceres, puesto que, de acuerdo a los diálogos continuos con ellas, se interviene el problema ya sea con la familia, la escuela o el contexto. (Entrevista 6).

Otro de los datos importantes que se encontraron durante este estudio es el hecho de que son varias las actividades que se integran para tratar de prevenir el riesgo no solo psicosocial, sino también físico de los estudiantes. La siguiente persona entrevistada expresa:

Se realizan talleres de convivencia en el aula, la cafetería que permite sólo opciones de alimentación saludable. Se hace también el proyecto de sexualidad, que no sólo interviene estudiantes, sino padres de familia, el de bienestar que se hace internamente, además el de abra palabra, que es el proyecto de lectura. En cuanto a la prevención de desastres actualmente se trabaja desde el área de ciencias naturales, donde se adecua y se conoce la ruta de evacuación. (Entrevista 8).

Como complemento a las anteriores actividades, algunos EE utilizan instrumentos para mantener actualizada la información de los estudiantes que pueden estar en cualquier tipo de riesgo psicosocial y por ello utilizan una ficha para mantener información del estudiante:

[...] existe la FARDA (Ficha de Relación Docente Estudiante), donde se construye a medida que pasan los días y las relaciones con ellos, de modo que se tenga información sobre el contexto: de donde viene el estudiante, la adaptabilidad a institución educativa, su crecimiento, fortalezas o procesos académicos y además la parte psicológica. (Entrevista 4).

Las estrategias anteriores no tendrían sustento ni serían factibles sin que existiera una orientación previa o marco legal que las fundamentara. Es por ello que varias instituciones encuentran en la legislación una guía adecuada para llevar a cabo sus actividades de prevención del riesgo, como lo encontrado en la entrevista 3, que, a través de su representante, dice: "Se hace uso de la ley 1620, donde los ubica en la ruta de atención, ya sea con sus padres de familia u acudientes, de ahí, se hace lo necesario desde la IE, y también con el Cerca, que la comunidad utiliza". 


\section{La gestión comunitaria y su aporte al mejoramiento académico pedagógico de los establecimientos educativos (EE)}

Ahora bien, surge el interrogante: ¿cómo la gestión comunitaria le aporta al mejoramiento académico pedagógico de los establecimientos educativos (EE) de la ciudad de Medellín para aumentar su calidad educativa?

Una primera respuesta parte de tomar experiencias positivas de la misma comunidad e implementarlas en los EE. Dice al respecto uno de los participantes de las entrevistas:

Fuimos la primera institución que hablamos de gestores estudiantiles, fuimos los primeros que hablamos de mediadores, los primeros en la ciudad; por qué, porque yo venía de hacer un trabajo social en el sector de Belén, entonces yo encontraba que ese trabajó que hacía dentro de las comunidades lo podíamos hacer dentro de las instituciones entonces empezamos a hacer gestores de la convivencia escolar [...] (Entrevista 10).

Otra de las respuestas surge de las alianzas que hacen los EE con otras entidades, un ejemplo lo presenta el entrevistado 6: "Sí, es que la Yamaha dijo: 'el proyecto de ustedes, venga hermano que eso está muy bueno allá', entonces, vienen trabajando con nosotros, entonces nos puso un profesor, y ahí vamos creciendo con la Yamaha".

Por otra parte, estas alianzas han propiciado la construcción de proyectos conjuntos. Como bien lo dice uno de los participantes:

Con las juntas, con lo de la Secretaría de la Mujer al hacer los convenios con las juntas comunales que ellos son los que presentan las propuestas y es el recurso de Pp, del proyecto participativo, aledaño claro el núcleo entero pues todos los colegios que están alrededor tienen algo en común con la Escuela Normal, la mayoría son egresados de acá, están, y tenemos un contacto directo en todo momento. (Entrevista 4).

También, el fortalecimiento y la pertinencia al contexto han favorecido la mejora de los procesos académicos pedagógicos de los EE, en este caso, la convivencia. Al respecto dicen: "Es muy extraño que los estudiantes vivan en un entorno más lejano del colegio, entonces eso hace que haya una identificación del entorno social y del ejercicio de convivencia que queremos facilitar y proveer en el colegio [...]" (Entrevista 1).

La alianza con las universidades también ha enriquecido los procesos pedagógicos, sobre este asunto se habla en la entrevista 4, refiriéndose a los logros pedagógicos aportados por la Universidad de Antioquia: 
Ellos también hacen una intervención muy bonita acá desde el proyecto de la universidad de Antioquia, porque interviene la parte pedagógica con estudiantes de la Antioquia con estudiantes de la licenciatura y también con los médicos, ese es otro de los proyectos que se tiene acá (Entrevista 4).

En este mismo sentido, los convenios y actividades de cooperación con otras entidades, en este caso con entidades internaciones, también han logrado impactar los procesos pedagógicos de los eE de la ciudad. A propósito, se dice:

Este convenio internacional permitió no solo conocer un nuevo modelo pedagógico, sino una transformación de la comunidad, en la que se involucra no sólo a los estudiantes, sino padres de familia y maestros, invitándolos a crecer en competencias, a saber, hacer y saber ser. (Entrevista 5).

De este modo, se observa que la gestión que hacen los establecimientos educativos con la comunidad, el entorno y la sociedad, les ha aportado ideas, proyectos, estrategias para la convivencia y alternativas de mejoramiento continuo de los procesos académicos pedagógicos que van "dirigidos al mejoramiento de la calidad de vida, así como también el avance de la comunidad educativa hacia la inclusión, participación, convivencia armónica, y proyección hacia el futuro" (Mamani y Machicado, 2012, párr. 11).

\section{Conclusiones}

Llegados a este punto se puede terminar diciendo que la concepción de la gestión comunitaria que tiene las instituciones estudiadas es amplia y determinada por cada uno de los contextos. Su implementación es guiada y motivada por las necesidades particulares de cada una de las comunidades en las que están insertas. Estas concepciones en su conjunto permiten entender e interpretar este concepto de forma variada, en cuanto se relacionan y construyen de acuerdo con la experiencia propia de los Ee y de sus participantes. En lo que respecta a las prácticas asociadas a la gestión comunitaria, se puede concluir que las instituciones tienen buenas estrategias para leer el entorno y reforzar la pertinencia al contexto, son también capaces de capitalizar dicha lectura y desde allí proponer actividades de proyección que tengan como propósito la construcción y rehabilitación del tejido social. De este modo, cabe decir que el sentido de pertenencia es alto. 
Tomando el caso de los egresados, se concluye que hay muy poco trabajo con estos: hay varias instituciones que no tienen ningún tipo de programa dirigido a esta población. La estrategia que más utilizan las instituciones para saber cómo están consiste en mantener información sobre el acceso de estos a la educación superior y el ingreso la universidad, las reuniones de integración realizadas por medio de jornadas deportivas y eucaristías. Otras instituciones presentan prácticas innovadoras en el trabajo con egresados: debido a que la población que atienden es de bajos recursos y presenta problemas para entrar en la vida laboral, se les presentan portales y ofertas de empleo de la ciudad, se les da información que mejore sus oportunidades, se les certifica en competencias; les informan donde están las canastas para el empleo, se les indica cómo hacer las hojas de vida y a dónde pueden Ilevarla. Se evidencian muchas actividades realizadas para los egresados en los EE, las cuales generan en ellos un alto sentido de pertenencia hacia su institución. Así, las instituciones educativas siguen contando con el aporte de los egresados en la gestión de los procesos educativos, lo que ayuda a mejorar la calidad educativa.

Es fundamental, entonces, fortalecer el proceso de seguimiento de los egresados a la función que tienen ellos en el contexto, ya que, si bien los EE de la muestra han demostrado que tienen estrategias para cumplir responsablemente con el proceso de seguimiento, hay que dejar claro que sigue siendo el punto de quiebre de los EE, puesto que es complicado la recolección de información y el seguimiento constante de estos.

Sobre las relaciones interinstitucionales de los ex de la ciudad, se puede resaltar la capacidad que tienen para construir alianza con diferentes instituciones. Se destacan las alianzas que se establecieron con entidades del contexto local, pero solo en el caso de una de las instituciones se han establecido relaciones internacionales, específicamente con FS España, FBC, Imagine y la Universidad de Texas; además, una de sus sedes es fruto de cooperación internacional con organizaciones de Puerto Rico.

Por último, y con relación al impacto que ha tenido la gestión comunitaria en el mejoramiento académico-pedagógico de las EE, se finaliza diciendo que a partir del conocimiento de otras experiencias exitosas, en el mismo tipo de contextos, las instituciones educativas pueden tomar experiencias para aplicarlas, en cuanto les ayuda a darse cuenta qué está pasando con la educación en otras instituciones que están en proceso de mejoramiento de su calidad, puede tornarse en un factor motivante y muy importante para los docentes y directivos, que también quieren lograr mejor calidad en contextos difíciles. La gestión comunitaria le permitió a los EE darse cuenta que debían fortalecer más el liderazgo. Por otra parte, motivó a muchas instituciones a cambiar sus modelos pedagógicos: permitirles poner en práctica la participación, hacerles saber en que era necesario modificar el manual de convivencia, qué mejoras tenían que hacerle desde 
el proyecto educativo institucional (PEI), como instrumento de concertación, de conciliación de prevención y como herramienta construida a partir de los propios principios, necesidades y acciones de cada establecimiento.

Así que la gestión comunitaria permitió a los EE informarse sobre cómo solucionar sus propios problemas de convivencia a partir de las respuestas que han dado a estos problemas otras instituciones con las que han entablado alianzas. Por otra parte, les ha permitido conocer procesos que hacen algunas instituciones en su día a día para solucionar problemas sociales y económicos, procesos y actividades que no se creían posibles. La gestión de la comunidad se constituyó en una fuente de metodologías, proyectos y modelos educativos que podrían ensayarse con miras a la adaptación de los establecimientos educativos a sus comunidades.

\section{Referencias}

Alcaldía de Medellín. (2012). Premios a la calidad de la educación, maestros para la vida. Medellín: Proantioquia.

Alcaldía de Medellín. (2013). Premios a la calidad de la educación, maestros para la vida. Medellín: Proantioquia.

Alcaldía de Medellín. (2014). Medellín construye un sueño maestro: Guía de postulación premios Ciudad de Medellín a la calidad de la educación. Medellín: Secretaría de Educación.

Avella, L. (2004). Manual de gestión comunitaria. Bogotá: Centro de Arbitraje y Conciliación de la Cámara de Comercio de Bogotá.

Institución Educativa Chachagüí. (s. f.). Gestión comunitaria. Recuperado de http://educhachagui.blogspot.com.co/p/gestion-comunitaria.html.

Jaramillo, R. (1999). La calidad de la educación: hacia un concepto de referencia. Revista Educación y Pedagogía, 16, 38, pp. 93-100. Disponible en http://tesis.udea.edu.co/bitstream/10495/3078/1/JaramiIloRodrigo_2004_calidadeducacionconceptorefencia.pdf.

Lepeley, M. (2002). Gestión de la calidad en educación superior: condiciones para maximizar el beneficio de la globalización. Pensamiento Educativo, 31, 203-235. Recuperado de http://pensamientoeducativo.uc.cl/index.php/pel/article/view/235/505.

Mamani, B. y Machicado, J. (2012). Gestión educativa comunitaria en Escuela Superior de Formación de Maestros de Warisata (2012). Recuperado de http://investigacioneducativawarisata.blogspot.com. co/2012/09/normal-0-21-false-false-false-es-x-none.html.

Ministerio de Educación Nacional. (2010). Revolución educativa 20022010: acciones y lecciones. Recuperado de https://www.mineducacion.gov.co/1759/articles-242160_archivo_pdf.pdf 
Martínez, A., Gutiérrez, C., Cardona, E., Guisao, L., Sierra, M. y Gómez, M. (2012). Articulación de la gestión educativa (directiva, académica, comunitaria, y administrativa financiera) en la institución educativa rural José Félix de Restrepo (Proyecto de trabajo de grado para optar al título de Especialista en Gerencia Educativa). Facultad de Educación, Universidad católica de Manizales. Manizales.

Palma, E. (2008). Percepción y valoración de la calidad educativa de alumnos y padres en 14 centros escolares de la región metropolitana de Santiago de Chile. Reice. Revista Iberoamericana sobre Calidad, Eficacia y Cambio en Educación, Educación, 6(1), 85-103.

Rodríguez, W. (2010). El concepto de calidad educativa: una mirada crítica desde el enfoque histórico-cultural. Revista Electrónica Actualidades Investigativas en Educación, 10(1), 1-22. Recuperado de http://www. redalyc.org/pdf/447/44713068015.pdf.

Secretaría de Educación de Medellín, Proantioquia, Universidad de Antioquia, Universidad Pontificia Bolivariana, la Universidad San Buenaventura Seccional Medellín, la Fundación Cinde y Universidad Católica Luis Amigó. (2014). Acta de compromiso. Medellín: Alcaldía de Medellín.

Sierra, F., Serna, C. y Pérez, E. (2002). Los padres de familia en las instituciones educativas de Medellín. Medellín: Corporación Región.

Taylor, S. y Bogdan, R. (1994). Introducción a los métodos cualitativos de investigación. Barcelona: Paidós.

Vásquez, T. (2013) Calidad y calidad educativa. Investigaciones Educativas, $17(2), 49-71$. 